\title{
DESARROLLO DE LAS HABILIDADES COGNITIVAS PROPIAS DE LAS ARTES ESCÉNICAS EN EL AULA
}

\author{
DESENVOLVIMENTO DAS COMPETÊNCIAS COGNITIVAS PRÓPRIAS DAS \\ ARTES CÊNICAS NA SALA DE AULA
}

\section{DEVELOPMENT OF COGNITIVE SKILLS OF THE PERFORMING ARTS IN THE CLASSROOM}

\author{
Marina BARBA DÁVALOS ${ }^{1}$
}

RESUMEN: La memoria, la atención, la escucha activa y el trabajo en equipo son habilidades que se desarrollan a un alto nivel en las artes escénicas. Los bailarines son capaces de recordar coreografías, sus movimientos, sus posiciones y desplazamientos en el espacio, a la vez que escuchan y siguen la música y al resto del cuerpo de baile. Un músico, tanto si memoriza como si lee la partitura previamente estudiada, mientras la interpreta satisfaciendo las exigencias técnicas y expresivas, está atento al resto de los miembros de la orquesta y al director. Los actores expresan el significado de un texto memorizado mientras interactúan con el resto del elenco, con los objetos escenográficos y con el propio espacio escénico. El objetivo de esta investigación es, a partir del análisis de las estrategias de aprendizaje y actuación de las diferentes disciplinas, proyectar una metodología de aplicación en el aula que permita desarrollar estas habilidades.

PALABRAS CLAVE: Música. Danza. Teatro. Habilidades cognitivas y no cognitivas. Educación.

RESUMO: A memória, a atenção, a escuta ativa e o trabalho em equipe são habilidades exercidas em alto nivel nas artes cênicas. Os dançarinos são capazes de lembrar coreografias, seus movimentos, suas posições e deslocamentos no espaço, ao mesmo tempo que ouvem e acompanham a música e o resto do corpo de baile. Um músico memoriza ou lê a partitura, previamente estudada, enquanto a executa, atendendo aos requisitos técnicos e expressivos: ele está atento ao resto dos membros da orquestra e ao maestro. Os atores expressam o significado de um texto memorizado enquanto interagem com o resto do elenco, com os objetos cenográficos e com o próprio espaço cênico. O objetivo deste artigo é, a partir da análise das estratégias de aprendizagem e ação das diferentes disciplinas, projetar uma metodologia de aplicação em sala de aula que permita o desenvolvimento dessas habilidades.

PALAVRAS-CHAVE: Música. Dança. Teatro. Habilidades cognitivas e não cognitivas. Educação.

ABSTRACT: Memory, attention, active listening and teamwork are skills exercised at a high level in the performing arts. Dancers are able to remember choreographies, their movements,

${ }^{1}$ Universidad de Alcalá (UAH), Guadalajara - España. Profesora Asociada del Departamento de Ciencias de la Educación. ORCID: https://orcid.org/0000-0001-8370-3569. E-mail: marina.barba@uah.es

RIAEE - Revista Ibero-Americana de Estudos em Educação, Araraquara, v. 15, n. esp. 4, p. 2727-2740, dez., 2020. e-ISSN: 1982-5587 
their positions and displacements in space, while listening to and following the music and the rest of the dance body. A musician memorizes or reads the score, previously studied, while executing it, meeting the technical and expressive requirements: being attentive to the rest of the orchestra members and the conductor. The actors express the meaning of a memorized text while interacting with the rest of the cast, the scenographic objects and the scenic space itself. The aim of this paper is, from the analysis of the learning and action strategies of different disciplines, to design a methodology of classroom application that allows the development of these skills.

KEYWORDS: Music. Dance. Theater. Cognitive and non-cognitive skills. Education.

\section{Introducción}

La memorización de una música, una coreografía o un texto dramático es tan sólo el primer paso, la base a partir de la cual se busca la perfección interpretativa de manera que el espectador disfrute del espectáculo ajeno a la sucesión de notas, pasos o palabras que lo conforman. Aunque las estrategias de aprendizaje son distintas en cada disciplina, los tipos de memoria y la forma de almacenar la información para automatizarla, son comunes para actores, músicos y bailarines.

Los siete tipos de memoria musical según (BARBACCI, 1965) son extensibles a la danza y al arte dramático. La memoria muscular en músicos y bailarines se encarga de automatizar movimientos discretos que responden a exigencias técnicas. En el caso de los actores se ha demostrado que recuerdan mejor su texto si relacionan sus intervenciones con movimientos, que no han de concordar literalmente con la palabra (NOICE; NOICE, 2001). La memoria auditiva proporciona al intérprete dramático y musical la capacidad de discernir y estimar su ejecución mientras que para el bailarín, la sola escucha de la música puede recordarle el movimiento. Asimismo, hay que considerar la importancia que presenta la memoria auditiva para los músicos que "tocan de oído". La memoria visual es común a músicos y actores en lo que respecta al aprendizaje de la partitura o al texto, mientras que los bailarines la utilizan para recordar los movimientos del coreógrafo. Músicos y bailarines comparten también la memoria visual cuando la emplean para recordar posiciones en el instrumento o posiciones corporales que, en el caso de los bailarines, recuperan de su imagen en el espejo. La memoria verbal almacena los textos dramáticos con exactitud, así como la terminología concreta de un paso o una nota. La memoria rítmica estructura y distribuye los movimientos de músicos y bailarines dentro de un tempo determinado y es subyacente en la cadencia de un texto sobre todo si está versificado. Los bailarines resultan exhaustivos en este procedimiento de memorización ya que cuentan los pulsos de la música, los distribuyen en compases y asignan a cada movimiento un 
número de ese cómputo musical. La memoria analítica recaba la información que se obtiene del análisis formal de una partitura, de un texto o de una coreografía. Y, por último, la memoria emotiva, imprescindible en las tres disciplinas, que recoge las características y matices interpretativos definidos, escogidos y meditados durante el estudio o ensayo.

La forma de almacenar la información según patrones que se van elaborando con el conocimiento de una competencia así como la eficacia y rapidez de los códigos de acceso y recuperación de esa información de la memoria a largo plazo, se corresponde con la Teoría de la Memoria Experta (WILLIAMON; EGNER, 2004). Los patrones en músicos y bailarines son una asociación sonoro-motora, mientras que según (NOICE; NOICE, 2001), en los actores el proceso es externo. En el análisis del texto se distinguen intenciones dramáticas, se les asigna una unidad de almacenamiento y se trabajan durante los ensayos en los que el actor debe implicarse motora y emocionalmente.

A medida que progresa el proceso de memorización, bien sea en el estudio individual o en los ensayos, se produce una apertura atencional. Sin perder la focalización en la tarea principal monitorizándola, el intérprete presta atención a más de un estímulo o actividad al mismo tiempo. Los actores interactúan con el resto del elenco, con los objetos escenográficos y con el propio espacio escénico; los músicos siguen al director y escuchan las diferentes voces musicales sincronizándose con ellas; y los bailarines toman conciencia de sus posiciones y desplazamientos espaciales a la vez que se integran en el cuerpo de baile. La conciencia de grupo y el trabajo en equipo resultan imprescindibles para alcanzar la perfección interpretativa.

Cuando se llega al escenario, todos estos aspectos están tan interiorizados al haberlos trabajado exhaustivamente durante los ensayos que, los intérpretes están preparados para solucionar cualquier fallo humano que surja durante la actuación sin que el público perciba ninguna anomalía. La capacidad de procesar información de forma rápida y automática, así como la capacidad de adaptación a situaciones cambiantes son otras de las habilidades que se fomentan con la dedicación a las artes escénicas.

La proliferación en los últimos años de investigaciones que desde el prisma de las neurociencias avalan el desarrollo del cerebro y de habilidades cognitivas a partir de las artes escénicas, sobre todo la música, sirven de argumento para defender su inclusión en la educación (PEÑALBA, 2017). Sólo en el campo de la música podemos encontrar estudios que relacionan el aprendizaje de un instrumento con el desarrollo de habilidades como la percepción auditiva, la memoria visual, la planificación, la velocidad de procesamiento o la flexibilidad cognitiva (HILLE; SCHUPP, 2015); la comprensión y fluidez verbal, la cognición espacial, la flexibilidad cognitiva, la velocidad perceptiva, el razonamiento, la cognición numérica o la memoria 
(HELMBOLD; RAMMSAYER; ALTENMÜLLER, 2005). En particular esta última, la relación entre el desarrollo de la memoria y la música ha despertado gran interés entre los investigadores: (HANSEN; WALLENTIN; VUUST, 2012), (JAKOBSON; LEWYCKY; KILGOUR; STOESZ, 2008), (LEE; LU; KO, 2007), (RIBEIRO; SANTOS, 2012).

Los ejemplos de publicaciones neurocientíficas en los que se comparan habilidades cognitivas de músicos y no músicos son numerosos y, en mi opinión, generalistas en cuanto a la definición en sí de músicos ya que engloba desde niños que han asistido a clases de algún instrumento, adultos que en algún momento de su formación estudiaron música, estudiantes de conservatorio superior hasta músicos profesionales. Aunque este tema necesitaría una revisión cuidadosa podemos encontrar un análisis cuantitativo de las investigaciones neurocientíficas relacionadas con la música publicadas entre los años 2005 y 2010 en (PETERSON, 2011).

En el presente trabajo no se pretende tanto justificar la presencia de las artes escénicas en la educación por las habilidades cognitivas que desarrollan, sino poner a disposición de docentes y alumnado, una serie de actividades enfocadas a fomentar aquellas habilidades que desde la perspectiva neurocientífica y desde mi experiencia en las artes escénicas, se realzan. El germen de alguna de estas actividades proviene de los entrenamientos teatrales dirigidos por Hassane Kouyaté, actor de la compañía de Peter Brook, que posteriormente he adaptado a la docencia en el aula y he impartido tanto en cursos de formación de profesorado como en el grado en Ciencias de la Educación. Las propuestas poseen pinceladas musicales, dancísticas o teatrales enmarcadas siempre en un contexto lúdico.

Se proponen diez actividades y en cada una de ellas se indica para qué aspectos está destinada: memoria (auditiva, visual), atención (atención focalizada, atención dividida, inhibición y motorización), razonamiento (planificación, velocidad de procesamiento, tiempo de respuesta y flexibilidad cognitiva) percepción (percepción auditiva, reconocimiento, percepción visual, escaneo visual y percepción espacial) y coordinación.

Para el buen funcionamiento de las actividades es aconsejable que el número de participantes oscile entre 8 y 12 . Si bien no existe límite de edad superior para disfrutar de estas propuestas porque resultan accesibles para niños, jóvenes, adultos y mayores; la edad para empezar, aunque siempre hay excepciones y se pueden realizar adaptaciones, sería como pronto a los 7 años. 


\section{Actividades}

\section{Actividad I}

Atención dividida, inhibición, escucha activa en equipo y resolución de problemas.

Los alumnos se sitúan en una fila, uno a lado de otro pero sin contacto físico y la mirada al frente. Sin ninguna señal tienen que ir saliendo de la fila dando un paso al frente de uno en uno. Si sale más de uno a la vez todos regresan a la fila y comienza de nuevo la actividad. Una vez hayan conseguido salir todos de la fila sin coincidir se reanuda el juego y esta vez tienen que ir saliendo de dos en dos. Progresivamente irá aumentando el número de personas que tengan que salir a la vez, 3, 4, 5...hasta que consigan dar un paso al frente todos juntos. Con esta actividad se trabaja la escucha en grupo y se pone en práctica la premisa "no es quiero sino puedo". Es decir, para cualquier trabajo en equipo las decisiones o propuestas no pueden imponerse sin valorar previamente si el ambiente del conjunto es acorde con la proposición. En esta actividad si no se ha escuchado previamente la disposición de los compañeros no se puede tomar la decisión unilateral de dar el paso al frente. Pero, a su vez, hay que tener la flexibilidad y la rapidez de actuar para que cuando, por ejemplo, la pauta es salir de tres en tres y se observa con la mirada periférica que solamente salen dos de la fila, incorporarse rápidamente en favor del equipo y el juego. De la misma manera hay que ser capaces de inhibir el impulso de salir de la fila si ya se han adelantado los participantes requeridos. Asimismo, hay que tener la capacidad de tomar decisiones y no esperar siempre a que otros den el paso adelante. En este juego se perfilan claramente los distintos roles que se suelen adoptar en los equipos: los que siempre están dispuestos a colaborar para resolver una situación, los que imponen sus opiniones sin escuchar al grupo, los que nunca arriesgan, los líderes que cuando toman una decisión cuentan siempre con el respaldo de todos y los que son capaces de evaluar el momento óptimo para lanzar su sugerencia a la vez que están siempre dispuestos a colaborar por el bien grupal.

\section{Actividad II}

Atención dividida, inhibición, escucha activa en equipo, percepción espacial, escaneo visual y memoria auditiva a corto plazo.

La primera fase de esta actividad es similar a la anterior solo que varía la distribución de los alumnos. Todos los miembros del grupo caminan por el aula ocupando todo el espacio y evitando la formación circular. De uno en uno y sin coincidir, se van deteniendo hasta que no quede nadie en movimiento. Una vez que la totalidad del grupo está inmóvil, sin ningún tipo 
de aviso previo, van reanudando la marcha de nuevo de uno en uno y sin coincidir con otro participante. A esta actividad en la que volvemos a trabajar la atención, la inhibición y la escucha en grupo, vamos a añadir la memoria auditiva a corto plazo, el escaneo y la percepción espacial. El mecanismo es el mismo: todo el grupo camina por el aula y cuando de uno en uno se van parando van diciendo objetos que hay en el aula sin repetir. Al retomar el movimiento de uno en uno, tienen que volver a enumerar los objetos no pudiendo mencionar el que ellos hayan dicho en la secuencia anterior.

De esta forma desarrollan el escaneo visual al buscar objetos del entorno, amplían la percepción visual al tener que estar atentos a los movimientos de sus compañeros a la vez que localizan los objetos y ocupan todo el espacio del aula evitando la tendencia a caminar en formación circular, incrementan la escucha del grupo para no tomar la decisión de detenerse o ponerse en marcha unilateralmente sin tener en cuenta al resto de los integrantes, aprenden a inhibir las acciones impulsivas que vayan en detrimento del equipo y ponen en funcionamiento la memoria para recordar los objetos que se han citado tanto para no repetirlos como para nombrarlos de nuevo al reincorporarse al movimiento.

Esta actividad se puede utilizar vehicularmente para el aprendizaje de contenidos de diferentes asignaturas. Por ejemplo, citando países sudamericanos durante la secuencia en la que se detienen y sus capitales cuando reanudan el movimiento, evitando que la misma persona diga la capital del país que anteriormente ha nombrado. Es aconsejable que el tema que se vaya a tratar no resulte tan complicado que los alumnos focalicen su atención en la materia y descuiden el resto de los aspectos comenzando a caminar en círculo, coincidiendo en la parada con otras personas, o repitiendo palabras que ya se han citado. Si esto ocurriera, se toma una opción más sencilla como puede ser citar palabras que comiencen por una letra determinada o enumerar objetos propios de un ambiente conocido, etc. Es importante que la temática escogida se les comunique cuando ya están caminando o cuando están todos en reposo para que no tengan tiempo de preparar su palabra, implicando así en la actividad la velocidad de procesamiento y el tiempo de respuesta.

\section{Actividad III}

Percepción auditiva, reconocimiento, atención dividida, velocidad de procesamiento y tiempo de respuesta.

Los participantes se sitúan en círculo. Sin un orden concreto cada uno de ellos va diciendo una palabra sobre una temática determinada, por ejemplo, animales mamíferos. Como 
en la propuesta anterior las temáticas pueden elegirse conforme a contenidos de otras materias pero siempre evitando que su dificultad anule el desempeño de la actividad. La serie se practica varias veces recordando el orden de intervención de cada participante hasta que se efectúa con cierta fluidez y velocidad. Comenzamos entonces con una nueva serie sobre otra temática, como puede ser, tipos de frutas. De nuevo, aleatoriamente, se establece el orden de intervención evitando que coincida con el de la serie anterior y se repite la secuencia hasta que se alcanza cierta destreza. Este es el momento en el que se recupera la primera serie para simultanear ambas. El objetivo es reconocer el estímulo, es decir, la palabra que precede nuestra intervención, y continuar la serie con la mayor rapidez. Al haber dos series en juego la atención ha de estar dirigida hacia dos estímulos distintos. Una vez que se ha alcanzado cierta comodidad y las dos series se mantienen durante un periodo de tiempo considerable con agilidad, añadimos otra nueva sobre otra temática diferente. La actividad no tiene un límite de series sino que dependerá de la disposición y características del grupo. Esta actividad, como el resto que aquí se plantean, no son un fin último sino un proceso de aprendizaje. El objetivo no es hacer el juego bien a la primera o juzgar a los participantes por sus errores, sino conseguir con la práctica y la repetición de las actividades que los rendimientos vayan mejorando. De hecho, una vez que el juego se realiza con comodidad debe ser descartado porque ya carece de interés. De esta manera, regresando a la actividad que nos ocupa, un grupo que al principio pueda presentar ciertas dificultades para mantener dos series simultáneamente, puede terminar compaginando cinco. Contémplese que según aumenta el número de series implicamos también la memoria al tener que recordar cada vez más palabras que actúan como estímulos a nuestra intervención. Es habitual que al aumentar la dificultad se genere tensión corporal en los participantes lo que resulta altamente contraproducente ya que instantáneamente se produce un bloqueo que inhibe cualquier destreza. Hay que controlar este efecto intentando relajar entre los juegos y creando un ambiente en el que se evite la frustración y los dictámenes entre participantes. Es preferible bajar la dificultad de la actividad antes de que el desánimo invada el aula.

\section{Actividad IV}

Percepción y memoria.

Esta actividad se realiza por parejas, uno actúa de guía y el otro permanece todo el tiempo con los ojos cerrados. El conductor pasea a su compañero cogiéndole de la mano por toda la sala y le va mostrando objetos y personas que tiene que reconocer por el tacto. Una vez concluido el recorrido, el participante que ha mantenido los ojos cerrados tiene que enumerar 
las personas y objetos que ha palpado. Es aconsejable que esta actividad la realice una sola pareja cada vez para evitar accidentes y para que el resto sea testigo de los aciertos en el reconocimiento. Una vez que todas las parejas han disfrutado de la experiencia se intercambian los roles. Con este juego fomentamos la confianza entre compañeros a la vez que activamos el reconocimiento táctil y ejercitamos la memoria.

\section{Actividad V}

Percepción y discriminación auditiva, inhibición y velocidad de procesamiento

Esta actividad es continuación de la anterior ya que se mantiene la pauta de trabajo en dúo donde uno de los participantes no puede utilizar el sentido de la vista. Cada una de las parejas va a elegir un sonido que puede ser, vocal, corporal, proceder de algún objeto o incluso, si se dispone en el aula, de algún instrumento musical, aprovechando así la actividad para reconocer y diferenciar diferentes timbres. Los participantes con los ojos cerrados se sitúan en la línea de salida de un trayecto lineal que tienen que completar. Es preferible que el desplazamiento se realice arrastrándose por el suelo para evitar percances. Cada uno de los participantes podrá moverse únicamente cuando suene el estímulo que anteriormente ha acordado con su pareja. Los compañeros que emiten el sonido no podrán hacerlo de forma continuada sino que tendrán que producir el estímulo de forma concisa y clara asegurándose en todo momento de que su compañero está recibiendo con precisión la señal a la vez que deben tomar la decisión adecuada de continuar o detener momentáneamente el estímulo para evitar que su compañero pueda colisionar con otros participantes. Para desarrollar la confianza en los dúos el conductor tiene que asumir la responsabilidad del bienestar de su compañero. Todas las parejas juegan a la vez y una vez completado el recorrido se intercambian los papeles. Con este juego vamos a desarrollar la percepción y discriminación auditiva a la vez que el guía desarrolla su velocidad de reacción y la inhibición de acciones que no resultan acordes con lo esperado al tener que adaptarse a un entorno cambiante.

\section{Actividad VI}

Velocidad de procesamiento y respuesta, inhibición, monitorización, memoria auditiva, percepción, reconocimiento y discriminación auditiva, planificación y flexibilidad cognitiva.

Siguiendo la línea de las dos actividades anteriormente propuestas, se mantiene la pauta de jugar por parejas con uno de los participantes con los ojos cerrados, pero esta vez la actividad 
la realiza una sola pareja en cada turno. Se construye un recorrido en el suelo con pequeños obstáculos que sortear (pasar por encima de una mochila o por debajo de una mesa) y direcciones diferentes que se puedan tomar evitando que la trayectoria sea lineal. Cada dúo diseña un código sonoro que responda a todos los movimientos que se han de realizar para completar el recorrido: avanzar, retroceder, girar a la izquierda, girar a la derecha, girar sobre sí mismo sin desplazarse hacia la derecha y hacia la izquierda, levantar una pierna para sortear un obstáculo, avanzar lateralmente hacia la derecha y hacia la izquierda, agacharse para pasar por debajo de un obstáculo, incorporarse o detenerse inmediatamente.

La elección del sonido que se va a asignar a cada movimiento es fundamental para realizar cómodamente el recorrido. La fuente de sonido tiene que ser fácil de diferenciar, la ejecución ha de ser simple para que sea precisa y el código completo ha de ser sencillo de memorizar para evitar confusiones tanto del emisor como del receptor. Cada dúo dispondrá de un tiempo para diseñar, memorizar y practicar el código y sus movimientos, primero con los ojos abiertos y luego cerrados. Cuando los participantes estén preparados se sitúa el receptor con los ojos cerrados en el encabezado del recorrido y el emisor en un punto fijo del aula que no podrá abandonar para dar ningún tipo de indicaciones.

Con esta actividad ambos están desarrollando la capacidad de planificación y la memoria auditiva. La persona que va a realizar el recorrido con los ojos cerrados va a potenciar su percepción auditiva al tener que reconocer los códigos sonoros y responder con el movimiento asignado. En algunas ocasiones, el simple hecho de cerrar los ojos y estar atento al estímulo auditivo, hace que el participante se desoriente, se paralice o empiece a confundir estímulos. En ese momento es fundamental la capacidad de reacción del emisor para resolver la situación y van a entrar en juego su velocidad de procesamiento y respuesta, su capacidad de inhibición y su flexibilidad cognitiva. Si el emisor se da cuenta de que el receptor ha confundido una señal con otra, podría variarla rápidamente adaptándose a la equivocación, o indicarle que se detenga inmediatamente dejar que se calme y proseguir pausadamente. En cualquier caso, tomar una decisión y monitorizarse para detectar si el error proviene de su emisión, ya que también puede confundir los códigos. Para evitar que se enfaden entre ellos, se echen la culpa uno a otro y en vez de solucionar aumenten la confusión, antes de empezar la actividad hay que subrayar que la resolución del conflicto necesita las dos partes. 


\section{Actividad VII}

Memoria, percepción y escaneo visual.

Esta actividad la realizan los participantes individualmente. Todos menos uno se colocan en una fila. El compañero que está fuera de la formación tiene que memorizar la posición de cada uno de los integrantes. Pasados unos instantes y fuera de la vista del observador, rehacen la formación con otro orden entre ellos. La misión del participante que está jugando es rehacer la fila con el orden primigenio. Si queremos relacionar esta actividad con el arte, en vez de formar una fila, se reproducirían los personajes y sus posiciones dentro de un cuadro determinado. De la misma manera, los personajes del cuadro intercambiarían sus papeles y posiciones y el participante observador tendría que recolocarlos es sus puesto originales. En ambos casos, con la fila o con el cuadro, se estarían trabajando la percepción y escaneo visual así como la memoria visual. Una vez que todos los alumnos hayan experimentado la actividad se podría aumentar su dificultad teniendo que reconstruir dos filas. Se distribuyen en la primera formación, el alumno que está fuera la memoriza, se reorganizan en una segunda fila que vuelve a ser memorizada y construyen una tercera con nuevas posiciones. El alumno tendrá que reconstruir primero la primera formación y a continuación la segunda.

\section{Actividad VIII}

Atención dividida y coordinación.

En esta actividad tienen que crear un cuento entre todos a la vez que van realizando una secuencia de cuatro movimientos: tumbados en el suelo boca arriba, sentados, tumbados boca abajo y de pie. Los movimientos de la secuencia se pueden variar para cada ocasión. Los participantes forman un círculo amplio en el que todos tengan contacto visual entre ellos y con el maestro de ceremonias, que es el papel que tiene que desempeñar uno de los alumnos escogido aleatoriamente y que irán acometiendo en las sucesivas historias otros compañeros. Todos tienen que seguir al maestro de ceremonias que irá cambiando de posición a su voluntad. Mientras, en un determinado punto del círculo se comienza a contar una historia que se irá transformando y creando a medida que vaya pasando por todos los participantes. En ningún momento se puede dejar de simultanear la historia con los movimientos de la secuencia y mucho menos en el turno de crear la historia. 


\section{Actividad IX}

Atención focalizada y memoria.

Los participantes se enumeran del uno al diez y se establece un ritmo base corporal para todo el conjunto: dos golpes en las piernas y dos palmadas, es decir, cuatro negras en un compás de cuatro por cuatro. Es importante que el ritmo sea sencillo para que el pulso se mantenga constante y no se acelere cuando se diversifique la atención. Los alumnos se desplazan por toda la sala, evitando caminar formando un círculo y efectuando este ritmo. En un determinado momento comienzan a llamarse de uno en uno con los números que previamente se han asignado de la siguiente manera: el número propio se hace coincidir con los golpes en las piernas y el número de la persona a la que se quiere llamar se dice a la vez que las palmadas. Por ejemplo, si el número 1 quiere llamar al 7 diría: "uno, uno, siete, siete”. Y el número llamado respondería llamando a su vez a otro: "siete, siete, dos, dos" y así sucesivamente hasta que alguien descuide su atención y falle. La persona que ha fallado se elimina sin decir qué número tenía, obligando al resto de los participantes a estar atentos y recordar los números de todos los compañeros y, sobre todo, los que se eliminan, ya que si al reanudar el juego se llama a un eliminado ese jugador también tiene que abandonar el juego. Como se puede contemplar, la dificultad aumenta de manera inversamente proporcional al número de participantes y de nuevo habrá que controlar el estado de tensión corporal que se hará evidente mediante un incremento de la velocidad y la intensidad del patrón rítmico. El aumento de estos dos parámetros complica a su vez la actividad porque, por una parte, supone una mayor velocidad de respuesta y, por otra, si el volumen crece excesivamente los alumnos dejan de escuchar claramente a quién están llamando.

\section{Actividad X}

Atención dividida, memoria y percepción auditiva.

Esta actividad requiere otras dos de preparación. En primer lugar vamos a trabajar la capacidad de escuchar y atender a más de una conversación a la vez. Un participante se sitúa en el centro y a cada lado dos personas empiezan a hablar libremente. Tras máximo un minuto, el participante situado en el centro tiene que contar todo lo que han ido relatando sus compañeros. Cuando todos han experimentado con dos conversaciones se va aumentando progresivamente el número de personas que hablan simultáneamente sin límite de conversadores. En esta actividad estamos desarrollando la atención dividida y la memoria para poder retener la información básica de cada uno de los conversadores. 
La segunda fase de la actividad consiste en mantener un diálogo rítmico por parejas. Uno de los dos propone una secuencia rítmica breve que el otro repite y a la inversa. En el siguiente turno el que acaba de repetir propone y su pareja imita. El resultado ha de ser un diálogo continuo sin pausas de dos personas proponiendo y repitiendo ritmos.

Una vez acometidas las dos fases anteriores los participantes se van a agrupar de cuatro en cuatro. Es aconsejable que los cuartetos se vayan sucediendo y que no realicen la actividad todos al mismo tiempo. Dentro del grupo, una pareja va a mantener un diálogo rítmico tal y como lo ha practicado con anterioridad, mientras que el objetivo de la otra pareja es interferir en esta comunicación hablando sin interrupción cada uno libremente. Tras máximo un minuto, la pareja que mantenía el diálogo rítmico tiene que ser capaz de relatar de qué estaban hablando sus otros dos compañeros. Si resulta muy complicado que hablen los dos oradores a la vez, primeramente se puede hacer con uno solo para ir incrementando el número a lo largo del tiempo. Todos los participantes han de probar los distintos roles para experimentar la diversidad estímulos que van a recibir. Por un lado los rítmicos, que van a recordar solamente el tiempo necesario para reproducirlos; y por otro lado la información de los conversadores que sí han de memorizar hasta que la actividad concluya. Además, tienen que simultanear esta diversidad de atención y memorización, con la creación de un nuevo ritmo cada vez que les toca proponer en el diálogo.

\section{Consideraciones finales}

Actualmente en España las artes escénicas no forman parte del currículo de Educación Primaria por lo que su aprendizaje y disfrute está ligado a una serie de condicionantes socioeconómicos del entorno del alumno: desde el interés de las familias hacia estas actividades extraescolares, cuestiones de índole logística hasta aspectos económicos que permitan la inversión en los materiales requeridos para su desempeño.

Las actividades propuestas pretenden compartir a través del juego las habilidades propias que se desarrollan con la práctica y aprendizaje de la danza, la música o el teatro con toda la comunidad educativa; a la vez que potenciar y reforzar estas competencias en los niños que sí cursan artes escénicas extraescolarmente.

La continuación en un futuro próximo de este trabajo sería implementar las actividades que aquí se han plasmado como una experiencia piloto en niños en edad escolar. Alcanzar acuerdos con instituciones públicas para realizar un estudio longitudinal que permita analizar el impacto de la intervención comparando el desempeño cognitivo antes y después de la 
estimulación. Para ello se invitaría a participar a todas las familias y niños de las instituciones determinadas estableciendo aleatoriamente un grupo de control y otro de intervención; y se desarrollaría una batería de pruebas cognitivas que nos sirvieran como instrumento de evaluación antes y después de la intervención.

Un resultado positivo abalaría la efectividad de la propuesta mientras que uno negativo respaldaría la necesidad de la presencia de las artes escénicas en la educación primaria para el desarrollo de ciertas habilidades cognitivas.

\section{REFERENCIAS}

BARBACCI, R. Educación de la memoria musical. Buenos Aires: Ricordi, 1965.

HANSEN, M.; WALLENTIN, M.; VUUST, P. Working memory and musical competence of musicians and non-musicians. Psychology of Music, v. 41, n. 6, p. 779-793, 2012.

HELMBOLD, N.; RAMMSAYER, T.; ALTENMÜLLER, E. Differences in primary mental abilities between musicians and non-musicians. Journal of Individual Differences, v. 26, n. 2, p. 74-85, 2005.

HILLE, A.; SCHUPP, J. How learning a musical instrument affects the development of skills. Economics of Education Review, Amsterdam, n. 44, p. 56-82, 2015.

JAKOBSON, L. S.; LEWYCKY, S. T.; KILGOUR, A. R.; STOESZ, B. M. Memory for verbal and visual material in highly trained musicians. Music Perception, v. 26, n. 1, p. 41$55,2008$.

LEE, Y.; LU, M.; KO, H. Effects of skill training on working memory capacity. Learning and Instruction, n. 17, p. 336-344, 2007.

NOICE, H.; NOICE, T. Learning dialogue with and without movement. Memory and Cognition, v. 29, n. 6, p. 820-828, 2001.

PEÑALBA, A. La defensa de la educación musical desde las neurociencias. Revista Electrónica Complutense de Investigación en Educación Musical, n. 14, p. 109-127, 2017.

PETERSON, A.D. The impact of neuroscience on music education advocacy and philosophy. Arts Education Policy Review, v. 112, n. 4, p. 206-213, 2011.

RIBEIRO, F. S.; SANTOS, F. H. Musical training and working memory span in beginners, veterans and with no musical knowledge children. Psicologia: Reflexão e Crítica, v. 25, n. 3, p. 559-567, 2012.

WILLIAMON, A.; EGNER, T. Memory structures for encoding and retrieving a piece of music: an ERP investigation. Cognitive Brain Research, n. 22, p. 36-44, 2004. 


\section{Cómo referenciar este artículo}

BARBA DÁVALOS, M. Desarrollo de las habilidades cognitivas propias de las artes escénicas en el aula. Revista Ibero-Americana de Estudos em Educação, Araraquara, v. 15, n. esp. 4, p. 2727-2740, dez., 2020. e-ISSN: 1982-5587. DOI: https://doi.org/10.21723/riaee.v15iesp4.14520

Remitido el: 10/09/2019

Revisiones requeridas el: 10/01/2020

Aprobado el: $30 / 04 / 2020$

Publicado el: 01/12/2020 